\title{
Radiometric calibration framework for ultra-high-resolution UAV-derived orthomosaics for large-scale mapping of invasive alien plants in semi-arid woodlands: Harrisia pomanensis as a case study
}

\author{
Madodomzi Mafanyaa,b,c, ${ }^{a}$, Philemon Tsele ${ }^{a}$, Joel O. Botaid, Phetole Manyama ${ }^{b}$, \\ George J. Chirima ${ }^{\mathrm{e}}$ and Thabang Monate ${ }^{\mathrm{c}}$ \\ aDepartment of Geography, Geoinformatics and Meteorology, University of Pretoria, Pretoria, South \\ Africa; \\ ${ }^{b}$ Biological Invasions Directorate, South African National Biodiversity Institute, National Botanical \\ Gardens, Pretoria, South Africa; \\ ${ }^{\mathrm{c}}$ GIS and Remote Sensing, CAD Mapping Aerial Surveyors, Pretoria, South Africa; \\ dSouth African Weather Service, Pretoria, South Africa; \\ eInstitute for Soil, Climate and Water, Geoinformatics Division, Agricultural Research Council, \\ Pretoria, South Africa
}

\begin{abstract}
Orthomosaics derived from consumer grade digital cameras on board unmanned aerial vehicles (UAVs) are increasingly being used for biodiversity monitoring and remote sensing of the environment. To have lasting quantitative value, remotely sensed imagery should be calibrated to physical units of reflectance. Radiometric calibration improves the quality of raw imagery for consistent quantitative analysis and comparison across different calibrated imagery. Moreover, calibrating remotely sensed imagery to units of reflectance improves its usefulness for deriving quantitative biochemical and biophysical metrics. Notwithstanding the existing radiometric calibration procedures for correcting single images, studies on radiometric calibration of UAV-derived orthomosaics remain scarce. In particular, this study presents a cost- and time-efficient radiometric calibration framework for designing calibration targets, checking scene illumination uniformity, converting orthomosaic digital numbers to units of reflectance, and accuracy assessment using in situ mean reflectance measurements (i.e. the average reflectance in a particular waveband). The empirical line method was adopted for the development of radiometric calibration prediction equations using mean reflectance values measured in only one spot within a 97 ha orthomosaic for three wavebands, i.e. red, green and blue of the Sony NEX-7 camera. A scene illumination uniformity check experiment was conducted to establish whether 10 randomly distributed regions within the orthomosaic experienced similar atmospheric and illumination conditions. This methodological framework was tested in a relatively flat terrain semi-arid woodland that is invaded by Harrisia pomanensis (the Midnight Lady). The scene illumination uniformity check results showed that at a $95 \%$ confidence interval, the prediction equations developed using mean reflectance values measured from only one spot within the scene can be used to calibrate the entire 97 ha RGB orthomosaic. Furthermore, the radiometric calibration accuracy assessment results showed a correlation coefficient $r$ value of $0.977(\mathrm{p}<0.01)$ between measured and estimated reflectance values with an overall root
\end{abstract}

${ }^{\star}$ Correspondence to: Madodomzi Mafanya; Department of Geography, Geoinformatics andMeteorology, University of Pretoria, Pretoria, South Africa. email: muzianho@gmail.com 
mean square error of 0.063 . These findings suggest that given the entire scene being mapped is experiencing similar atmospheric and illumination conditions, then prediction equations developed using mean reflectance values measured in only one spot within the scene can be used to calibrate the entire orthomosaic in semi-arid woodlands. The proposed methodological framework can potentially be tested and adapted for use in large-scale crop mapping and monitoring in precision agriculture, land-use/land-cover classification as well as plant species delimitation, particularly for mapping widespread invasive alien plants such as $\mathrm{H}$. pomanensis.

\section{Introduction}

Orthomosaics derived from consumer grade digital cameras on board unmanned aerial vehicles (UAVs) are increasingly being used for vegetation mapping (Laliberte et al. 2011; Pádua et al. 2017; Bonnet, Lisein, and Lejeune 2017). Recently, the UAV remote-sensing (UAV-RS) approach has been frequently reported as advantageous over spaceborne and traditional airborne remote-sensing approaches due to the resultant high temporal and spatial resolution data as well as the survey cost efficiency associated with this approach (Femondimo et al. 2011; Müllerová et al. 2017; Babapour et al., 2017). Applications of the UAV-RS approach include inter alia agriculture (Li et al. 2017), precision agriculture (Primicerio et al. 2012; Gómez-Candón, De Castro, and Lopez-Granados 2014; Rokhmana 2015; Bagheri 2017), land use (Akar 2017), forestry (Thiel and Schmullius 2017; Torrescan et al. 2017), archaeology (Rinaudo et al. 2012; Fernández-Hernandez González-Aguiler, Rodriguez-Gonzalvez, and Mancera-Taboada 2015), classification of native vegetation (Zhang 2014), and mapping of invasive alien plants (IAPs) (Dvořák et al. 2015; Müllerová et al. 2016; Hill et al. 2017).

UAV-RS imagery are highly influenced by prevailing environmental conditions at the time of data acquisition (Kelcey and Lucieer 2012; Yang et al. 2017). These environmental conditions include the atmospheric composition (e.g. water vapour and aerosols) as well as solar illumination patterns that depend on variations in the earth-sun geometry for different seasons and times of the day (Nguyen et al. 2015; Honkavaara et al. 2017). As a result, images of a target scene acquired by the same sensor on different seasons or times of the day may have different properties (Haghighattalab et al. 2016). On the other hand, images acquired by the same sensor may contain noise due to lens distortions, sensor systematic errors, as well as the variation of the camera sensitivity across the same image (Wang et al. 2006; Hugemann 2010; Del Pozo et al. 2014).

To have lasting quantitative value, it is standard practice to have remotely sensed data calibrated to physical units of reflectance (Smith and Milton 1999). The radiometric calibration process converts image digital numbers (DNs) to at-surface reflectance units so as to enable quantitative analysis across data acquired at different seasons or times of the day as well as by different sensors. Calibrated data sets may be cross compared because while image DNs of a target object change depending on environmental factors such as the incoming irradiance and atmospheric conditions (Honkavaara et al. 2013), 
after radiometric calibration, the spectral characterization of an object becomes possible (Kelcey and Lucieer 2012; Crusiol et al. 2017). Moreover, radiometric calibration improves the accuracy of derived vegetation indices as well as biochemical and biophysical parameters (Nguyen et al. 2015). Nevertheless, studies that applied the UAV-RS for IAPs monitoring made use of thematic image classification without either conducting geometric or radiometric calibration (Dvořák et al. 2015; Müllerová et al. 2016; Mafanya et al. 2017). This is because UAV-RS of biological invasions is optimized to be time and cost efficient so as to enable mapping of IAPs at the right phenological stage, at an affordable cost and with a quick turnover (Müllerová et al. 2017; Hill et al. 2017; Tesfamichael et al. 2017). However, monitoring of IAPs could encompass not only qualitative thematic mapping but also quantitative image analysis such as image band mathematics (e.g. vegetation indices) as applied inter alia in agriculture (Li et al. 2017), precision agriculture (Honkavaara et al. 2013; Candiago et al. 2015; Sonaa et al. 2016), and forestry (Thiel and Schmullius 2017; Torrescan et al. 2017). Since one of the aims in UAV-RS is cost reduction, a radiometric calibration framework for UAV-based IAPS monitoring ought to be cost and time efficient.

UAV-RS radiometric calibration poses different challenges to spaceborne and manned aircraft remote sensing. The low flight altitudes of mostly less than $200 \mathrm{~m}$ due to some governmental restrictions mean that for the same area of interest there will be more UAV-RS images acquired due to the associated small footprint than there would have been if the data was acquired from a satellite platform or from a piloted aircraft (Grenzdörffer, Engel, and Teichert 2008; Cracknell 2017). This means that it is not feasible to have at-surface in situ reflectance calibration measurements for all acquired UAVderived images as it would be impractical to place enough calibration targets on the ground for this goal. As a result, most radiometric calibration procedures were developed for a single image that is usually an aerial view of the calibration site. For instance, Taylor (2015) successfully demonstrated the process of radiometric calibration of a modified digital single-lens reflex camera using a single image while Kelcey and Lucieer (2012) used a single image to demonstrate the radiometric calibration workflow for a six-band UAV-borne multispectral sensor. Moreover, Wang and Myint (2015) used a single-sample image to demonstrate a simplified empirical line method (ELM) for radiometric calibration of a UAV-derived colour infrared image. Usually, the radiometric calibrated images are then mosaicked to produce a multi-band orthomosaic as done in Clemens (2012). However, based on the successful demonstrations of aforementioned studies, single-image radiometric calibration may become impractical for large-scale UAV-RS vegetation mapping. This is because it is not feasible to have calibration targets and at-surface reflectance measurements for every image captured even for small study areas (Hakala, Suomalainen, and Peltoniemi 2010).

IAPs tend to spread across very large areas ( $>90 \mathrm{ha}$ ), and this necessitates a radiometric calibration framework for large-scale mapping. For instance, the cactus plant Harrisia pomanensis (commonly known as the Midnight Lady) has been detected invading an area of not less than 10,000 ha in Limpopo, South Africa (Mafanya et al. 2017). Following the efforts made in image-by-image radiometric calibration studies (Taylor 2015; Kelcey and Lucieer 2012; Wang and Myint 2015; Crusiol et al. 2017), this study presents a cost- and time-efficient radiometric calibration framework for converting DNs of UAV-derived orthomosaics to physical units of reflectance for large-scale mapping of 
H. pomanensis in flat semi-arid woodlands. Included in the framework are procedures for calibration target design, scene illumination uniformity assessment, prediction equations derivation, and radiometric calibration accuracy assessment using in situ at-surface mean reflectance measurements (i.e. the average reflectance in a particular waveband) measured in only one spot within the scene.

\section{Vicarious radiometric calibration framework}

\subsection{Calibration target design}

For radiometric calibration, targets that are homogenous and resemble Lambertian properties are highly desirable (Del Pozo et al. 2014). While large homogenous natural targets are usually used for radiometric calibration of satellite imagery, use of artificial targets that are specifically designed for a project is usually made in UAV-RS. This is because coloured artificial targets are easy to transport to the field and can be easily moved to the next study area. Moreover, for the relatively small UAV-RS orthomosaics, compared to satellite RS scenes, it is often difficult to find naturally available pseudo-invariant features. The types of materials used in UAV-RS radiometric calibration target design include plywood (Kelcey and Lucieer 2012), Masonite hardboard (Wang and Myint 2015), polyvinyl chloride (PVC) sheets and canvas (Del Pozo et al. 2014; Crusiol et al. 2017). For radiometric calibration target design, this study made use of the relatively affordable Masonite hardboards for the radiometric calibration target and a reinforced PVC sheet for the check target (Figure 1). The reinforced PVC check target with red, green, blue, black, and white control colours was also placed in the study area for accuracy assessment and error analysis.

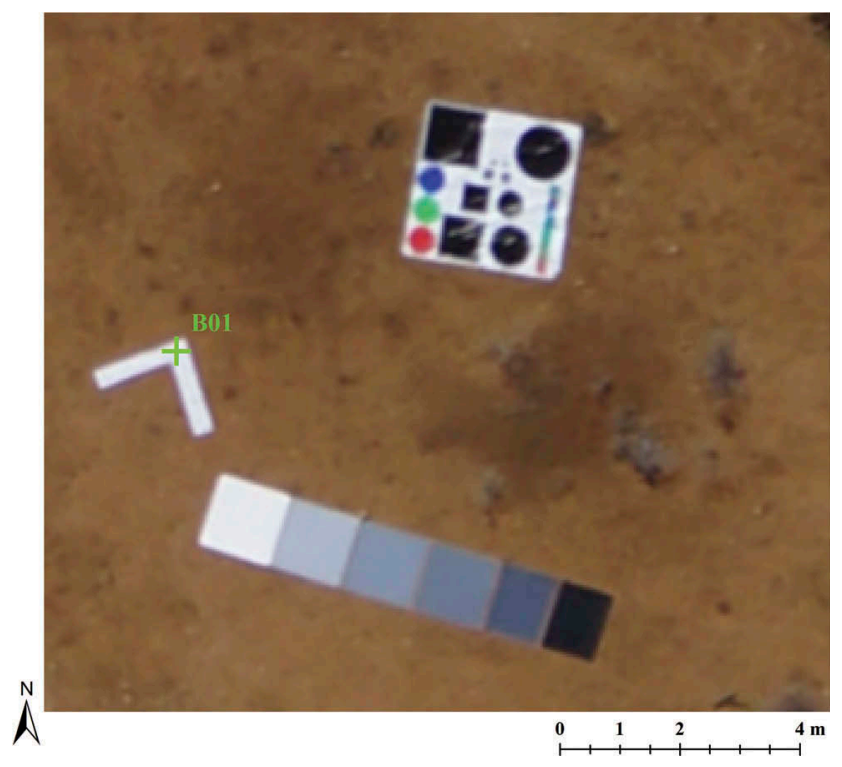

Figure 1. UAV aerial view of the control target (bottom) and check targets (top) taken by the Sony Nex-7 camera on board the UAV flown at a height of $160 \mathrm{~m}$ AGL. The L-shaped marker in the middle (B01) was considered to be receiving similar irradiance as the radiometric calibration and check targets due to spatial proximity. 


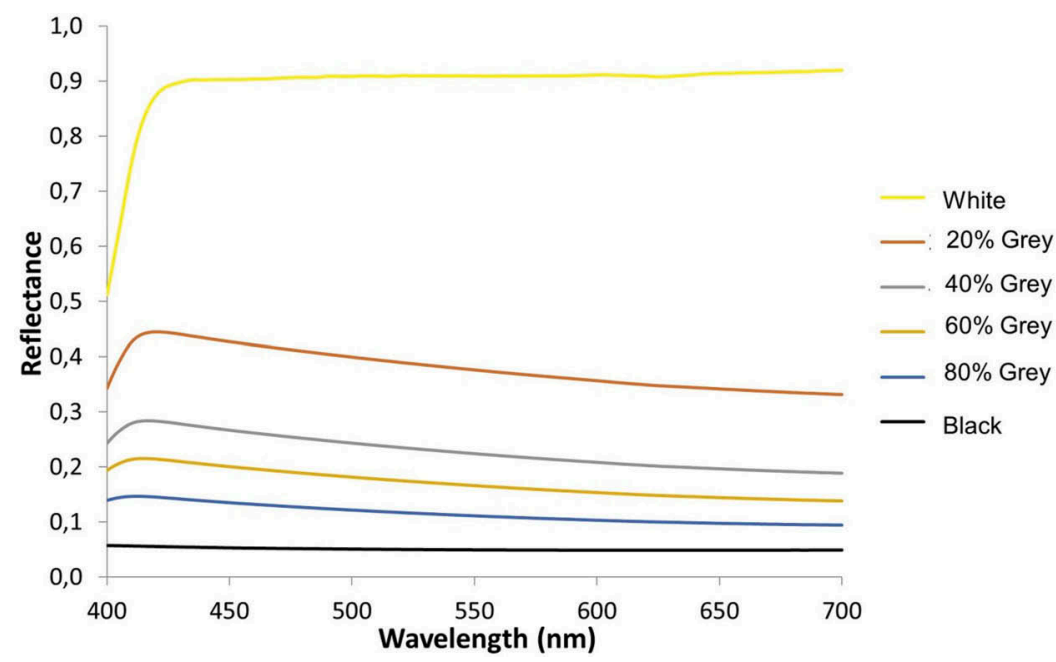

Figure 2. Mean spectral response curves of the Lambertian radiometric calibration targets. The white target (curve shown in yellow) has high reflectance while the black target has low reflectance.

With regards to the calibration target design, three standard size Masonite hardboards of $244 \mathrm{~cm} \times 122 \mathrm{~cm}$ were painted with $1 \mathrm{~mm}$ thick super white and black paints that were further mixed into four colour values of grey (totalling six calibration grey values). The calibration target was designed to form a grey gradient as follows: white, $20 \%$ grey, $40 \%$ grey, $60 \%$ grey, $80 \%$ grey and black. The mean spectral signature for each grey value is shown in Figure 2, which demonstrates the Lambertian properties of the created calibration target. The Lambertian properties of the grey gradient painted Masonite calibration target were first tested in a controlled room under an artificial light source, and this material was found to be highly Lambertian in the visible region of the electromagnetic spectrum as also demonstrated by Wang and Myint (2015).

Moreover, white PVC sheets were used to design L-shaped scene illumination uniformity check markers. The white PVC sheets were chosen to design the L-shaped markers due to ease of transportation and visibility in both raw images and the orthomosaic. The L-shaped PVC markers were placed randomly across the scene for orthorectification ground control points (GCPs) surveying and the scene illumination uniformity check experiment. The calibration targets were placed in the study area before the UAV flight and the capturing of their spectral signatures was done before, during, and after the UAV flight. This did not add significant time to the mapping process as the imagery was acquired at noon together with the spectral signatures. Also this was done so as to minimize the differences in solar illumination conditions between image data and in situ spectral signatures acquisition. It took almost 43 and 118 min to acquire image data and in situ spectral signature measurements, respectively.

\subsection{Scene illumination uniformity check}

Intuitively, a large-scale vicarious radiometric calibration method using radiometric calibration targets placed in only one region of the study area will require that the prevailing environmental conditions and solar illumination patterns (due to sun elevation angle) across the scene 


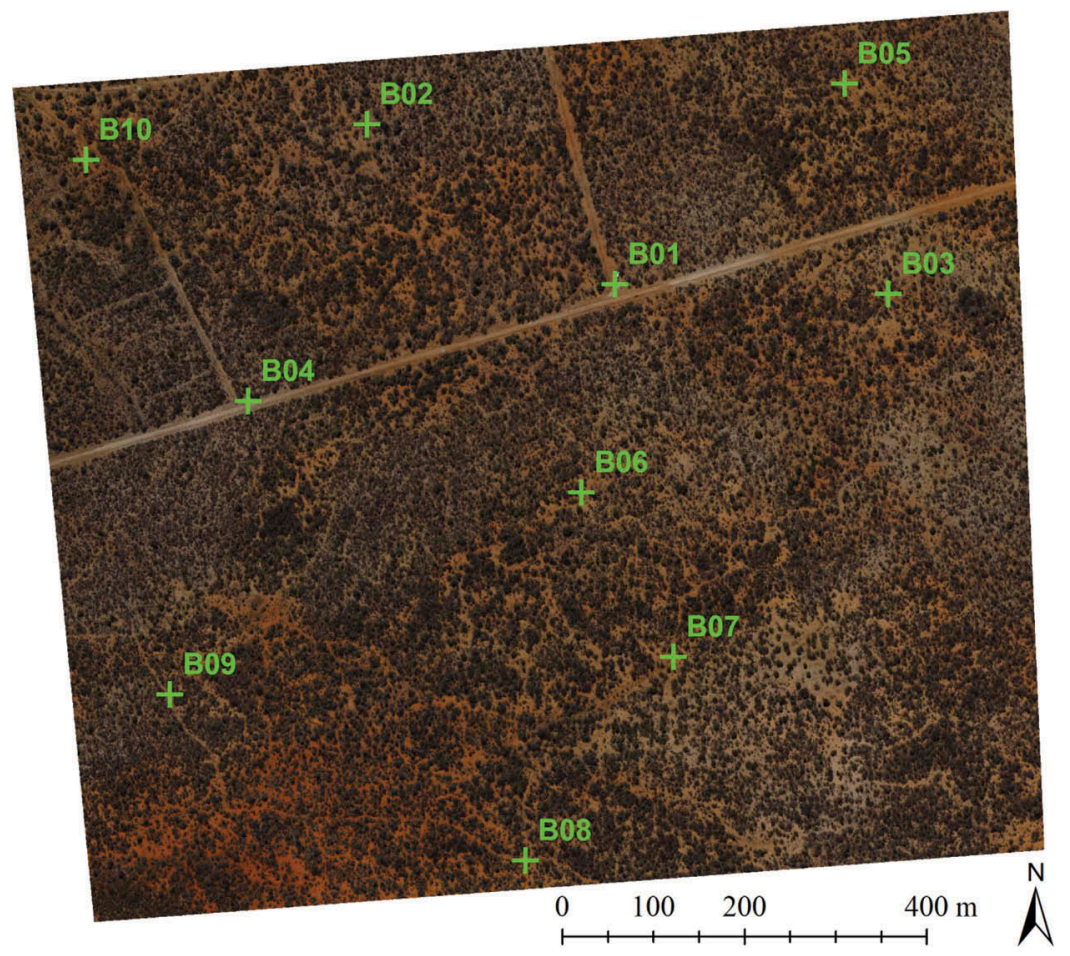

Figure 3. Mapped area showing the distribution of the 10 L-targets B01-B10. The target position is indicated by centres of the green crosses.

be uniform. To check whether the environmental conditions and solar illumination patterns are uniform across the scene, 10 L-shaped PVC markers were placed randomly, across the mapping area in this study (Figure 3). The mean DNs of the L-shaped markers, extracted from raw UAV images, were used to check whether the illumination patterns and prevailing environmental conditions across the scene were the same during image data acquisition, which took about $43 \mathrm{~min}$. Due to the $60 \%$ side and $80 \%$ forward overlap photo sampling method employed in this study, each of the L-shaped markers appeared in at least five raw images. The ArcGIS 10.4 Spatial Analyst Zonal Statistics tool was used to extract the targets mean pixel DNs from a total of 58 raw images. The B01 marker was considered the reference illumination check maker because it was the closest marker to the radiometric calibration target (Figure 1). A two-sample Student's $t$-test was conducted to check whether the mean DN of the B01 marker, appearing in 6 images, was the same as the mean DNs of the rest of the 9 other markers that appeared in the remainder 52 images. This scene illumination uniformity check experiment was deemed necessary because mean reflectance values measured in only one spot within the scene should be used to calibrate the entire orthomosaic if and only if the entire orthomosaic is experiencing similar atmospheric and illumination conditions.

\subsection{Empirical line method}

Vicarious radiometric calibration procedures mainly focus on modelling the relationship between image DNs and in situ at-surface reflectance values of the calibration targets. In 
particular, the ELM is used to obtain image reflectance based on the measured spectral signatures of calibration targets that are placed on the ground (Honkavaara et al. 2013). The ELM calibration prediction equations derived using mean target DNs and mean reflectance per waveband for each colour value of the calibration target were used in this study. These equations were derived using mean DNs and mean reflectance values measured from a target located at a single spot of the study area. For in situ spectral measurements, the Analytical Spectral Devices, Inc. FieldSpec 3 Pro calibrated spectroradiometer was used to take 25 spectral signatures (each spectra was a mean of 10 samples) for all the six grey values of the calibration target. An average of the 25 spectra was taken for each grey value, thus totalling 250 individual measured spectral signatures for each grey value.

To obtain a single in situ at-surface reflectance measurement for each grey value on the calibration target, the $1 \mathrm{~nm}$ spectral measurements were averaged according to the spectral response curve of the Sony NEX-7 CMOS sensor. Berra et al. (2015) measured the spectral sensitivity of the Sony CMOS using a monochromatic light source and the relative colour channels of the Sony CMOS were found to be approximately $400-490 \mathrm{~nm}$, $491-590 \mathrm{~nm}$, and $591-690 \mathrm{~nm}$ for the blue, green, and red colour channels, respectively. The same method was followed to get mean spectral signatures for the coloured PVC check targets. To obtain the mean image DN for each grey value of the calibration target, use of the Zonal Statistics tool within the ArcGIS 10.4 Spatial Analyst extension was made. Considerable care was taken to create polygons that contain only pixels that belong only to that particular grey value.

In most studies, a linear relation between image DNs and the at-surface reflectance values is assumed (Karpouzli and Malthus 2003; Staben et al. 2012). When more than two calibration targets are used, the relationship between image DN and reflectance does not have to be assumed to be linear (Smith and Milton 1999). In this study, the mean DNs were regressed with the mean reflectance values and the relationship between image DN and reflectance was found to be exponential for all the three image wavebands as also observed in Wang and Myint (2015). Following the simplified ELM proposed by Wang and Myint (2015), a natural log transformation was performed on all the six mean in situ at-surface spectra for each image waveband. The transformation showed that a linear relationship exists between mean image DNs and the mean natural log-transformed reflectance values for each waveband. When the $y$-intercept of the linear equation is converted back to reflectance, the result is a minimum at-surface reflectance value for that particular image waveband. Therefore, the $y$-intercept of each waveband is the constant radiometric calibration parameter for that particular waveband. Furthermore, the $y$-intercept coordinate can be used as an initial point in a Cartesian plane. To derive an ELM prediction equation, another point in the Cartesian coordinate system can be represented by the mean DN and mean at-surface reflectance of the entire calibration target for each image waveband. These two points can then be used to calculate the gradient $(m)$ of the calibration equation as shown in Equation (1):

$$
m=\frac{B_{y}-A_{y}}{B_{x}}
$$

where $A_{y}$ is the constant radiometric calibration parameter, $B_{x}$ is the mean DN of the calibration target, and $B_{y}$ is the natural log-transformed mean reflectance of the 
calibration target (Wang and Myint 2015). The developed ELM prediction equations were used to convert each waveband of the orthomosaic from DNs to reflectance values using the raster calculator in QGIS (Quantum GIS development team 2017). Subsequently, the three image wavebands were stacked together to give a colour RGB image with reflectance values instead of DNs. The radiometric calibration using the raster calculator in QGIS took about $30 \mathrm{~min}$ and therefore did not delay the mapping process.

\subsection{Radiometric calibration accuracy assessment}

The mean reflectance values of the PVC check target with black, blue, green, red, and white colours were used for accuracy assessment. During the acquisition of the in situ validation data, 5 spectra (a total of 50 individual spectral signatures) were measured for each check colour. The Zonal Statistics tool in ArcGIS 10.4 was used to get the mean reflectance of each coloured check target for each waveband. There were 6 available mean reflectance values for each coloured check target (i.e. 1 estimated and 1 measured mean value for each waveband thus totalling 24 values). The estimated image mean reflectance values and mean reflectance in situ measurements for each coloured target circle or square were then correlated so as to validate the large-scale radiometric calibration method used in this study. Error analysis through root mean square error (RMSE) computations was used to supplement the correlation analysis.

\subsection{Radiometric calibration process flow diagram}

This framework consists of data acquisition, derived products, and methodological phases (Figure 4). The first acquisition phase is the capture of GCPs that were observed at the randomly distributed L-shaped markers for orthorectification and the scene illumination uniformity check experiment. This is followed by the recording of spectral signatures of the calibration and the check targets. Target spectral signatures were recorded almost contemporary with the UAV flight mission (i.e. the UAV image acquisition phase). Only two final products are derived when using the proposed framework and those are (1) the raw orthomosaic in DNs and (2) the radiometric calibrated orthomosaic in physical units of reflectance. Furthermore, the image processing and statistical methods applicable to this framework are explained in the $H$. pomanensis case study.

\section{H. pomanensis case study}

\subsection{Study area}

The study area is located near the Alldays town within the Capricon district in the Limpopo province of South Africa (Figure 5). The area is characterized by (1) a semi-arid climate, (2) summer rainfall, and (3) average midday temperatures of $22.3^{\circ} \mathrm{C}$ and $31.9^{\circ} \mathrm{C}$ in winter (June-August) and summer (October-February) seasons, respectively (Mzezwa, Mlisi, and Van Rensburg 2010). The rainfall ranges from a few millimetre in winter and could escalate to a maximum of approximately $81 \mathrm{~mm}$ in summer (Mzezwa, Mlisi, and Van Rensburg 2010). The 97 ha mapped area is shown in Figure 5(b). The study area is a 


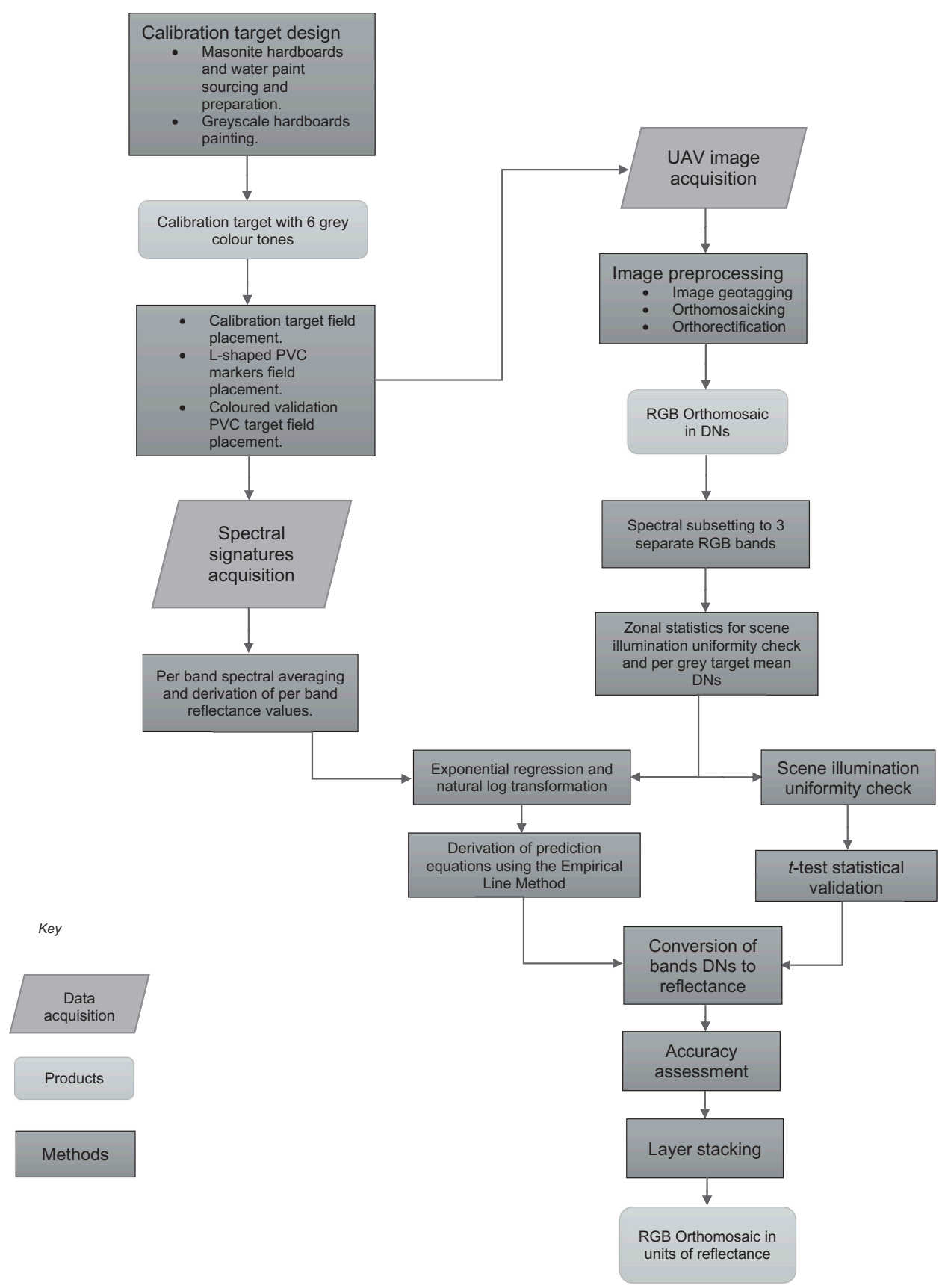

Figure 4. Proposed radiometric calibration framework for large-scale mapping of $H$. pomanensis in semi-arid woodlands using UAV-derived orthomosaics.

relatively flat terrain with orthometric height values ranging from 800 to $817 \mathrm{~m}$. Tree species that occur in this heterogeneous semi-arid woodland include Commiphora mollis (velvet-leaved corkwood), Commiphora neglecta (green-temmed corkwood), Commiphora schimperi (glossy-leaved corkwood), Commiphora viminea (zebra-bared 


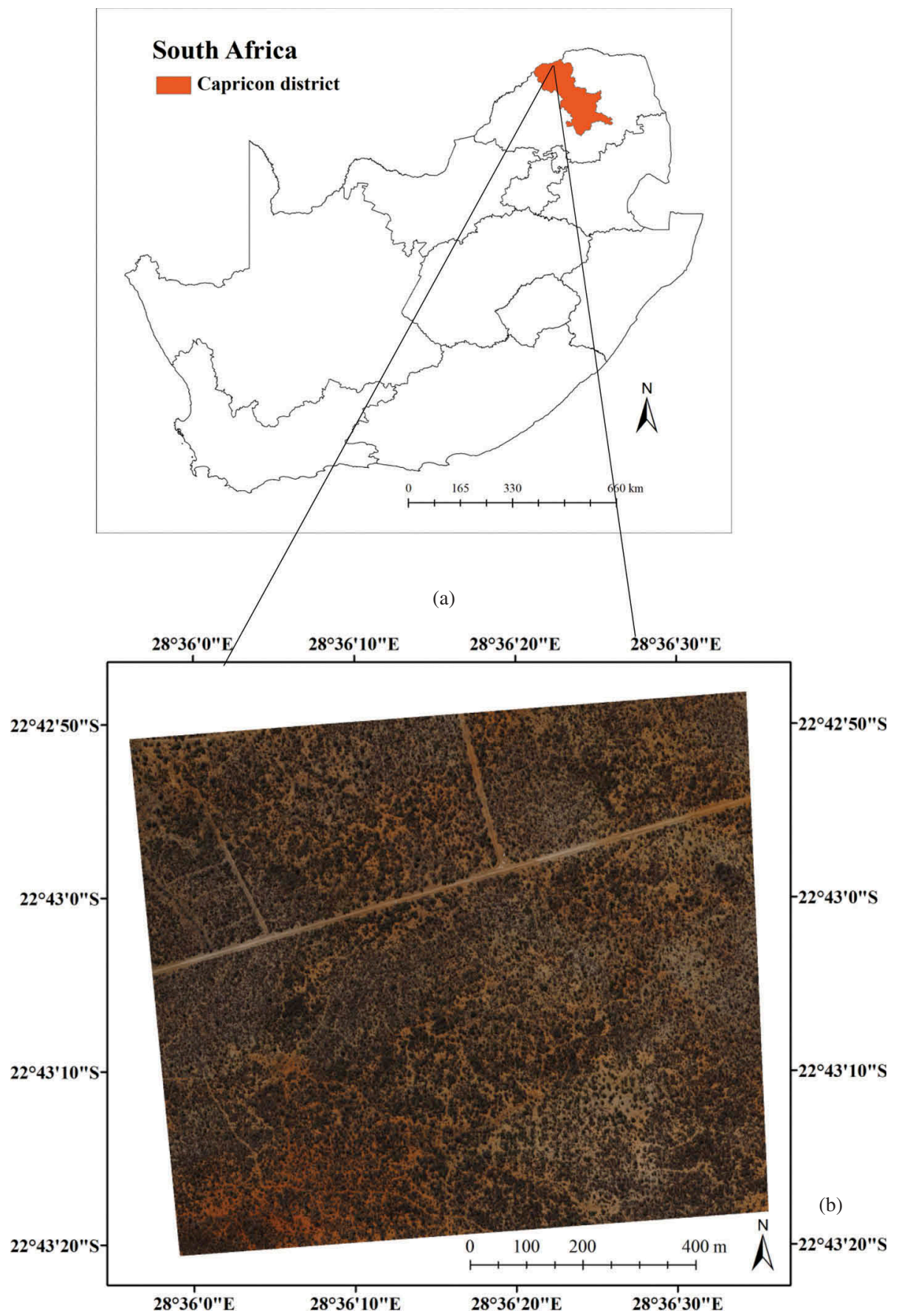

Figure 5. (a) Map of South Africa showing the location of Capricon district within the Limpopo province and (b) a subset of the UAV-derived ultra-high spatial resolution $(5 \mathrm{~cm})$ RGB orthomosaic.

corkwood), Acacia nilotica (scented pod thorn), Acacia tortilis (umbrella thorn), Acacia nigrescens (knob thorn), Acacia robusta (broad pod robust thorn), Acacia mellifera (black thorn), Balanites maughamii (green thorn), Albizia sp. (false thorn), Kirkia acuminata 
(white seringa), Combretum imberde (leadwood), Combretum hereroense (Russet bush willow), Boscia albitrunca (shepherd's tree), Berchemia zeyheri (red ivory), Maerua angolensis (bead bean), Lannea sp. (false marula), Sclerocarya birrea (marula), and Opuntia humifusa (devil's tongue), amongst others (Mafanya et al. 2017). The $H$. pomanensis invasive plant forms dense clusters in the described woodland area and could consequently replace pasture, injure animals, and thus inhibit cropping and grazing (Wilson et al., 2013). $H$. pomanensis is a succulent cactus with jointed spiny fleshy stems and thorny spikes, and when these stems touch the ground, they develop roots and spread. The plant seeds are spread by birds and monkeys that consume the red fruits of the plant. H. pomanensis was detected by the South African National Biodiversity Institute: Invasive Species Programme in 2011 as part of ongoing efforts at incursion response planning (Wilson, Panetta, and Lindgren 2016).

\subsection{Utilized UAV and digital camera}

To acquire ultra-high-resolution images (ground sample distance $<5 \mathrm{~cm}$ ) for mapping $H$. pomanensis, utility of a customized UAV with a net weight of $1.5 \mathrm{~kg}$ (excluding camera and batteries) and a payload of approximately $5 \mathrm{~kg}$ was made (Figure 6). The fundamental components of the utilized UAV included a Global Navigation Satellite System (GNSS) receiver, an Inertial Measurement Unit (IMU) sensor, and a barometer. The UAV was flown at a ground speed of $14 \mathrm{~m} / \mathrm{s}$ at $160 \mathrm{~m}$ above ground level (AGL). The camera mounted on the UAV was the Sony NEX-7, which has a Complementary Metal-Oxide Semiconductor (CMOS) imaging sensor. The Sony NEX-7 is a mirrorless interchangeable lens camera whose sensor format is the Advanced Photo System type-C CMOS. The camera exposure settings were set manually (as opposed to auto-exposure) so as to obtain equally focused images. The camera manual exposure program settings are shown in Table 1 and were established through a set of trial runs before being used

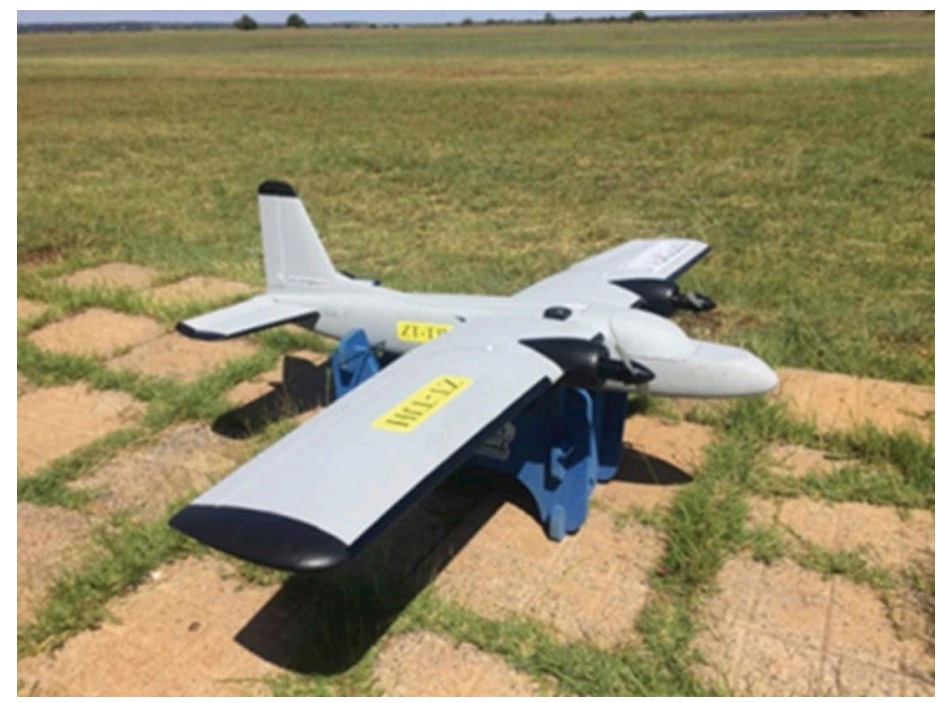

Figure 6. Utilized fixed wing UAV for autonomous image capturing. 
Table 1. Manual exposure program of the camera.

$\begin{array}{ll}\text { Exposure time } & 1 / 1000 \mathrm{~s} \\ \text { ISO-speed } & \text { ISO-100 } \\ \text { Focal length } & 16 \mathrm{~mm} \\ \text { Maximum aperture } & \mathrm{f} / 2.96875\end{array}$

in this case study. The resultant 611 24-bit single images had $6000 \times 4000$ pixels each with ground coverage of $234 \mathrm{~m} \times 156 \mathrm{~m}$. The camera parameters depend on the prevailing wind speed and flight height and thus should be customized accordingly.

\subsection{Image processing}

The images, GCPs, and spectral data were acquired on 12 August 2016. This month in particular is naturally known to be the South African late winter season and thus regarded as the most appropriate phenological stage for mapping $\mathrm{H}$. pomanensis (Mafanya et al. 2017). This is because the deciduous background vegetation shed leaves while $H$. pomanensis remains evergreen during this season. During image acquisition, the GNSS/IMU system on board the UAV was logging GPS coordinates of each captured image as events that were later used to geotag the raw images using Ardupilot's open source Mission Planner (Osborne 2016). The side and forward overlap were set to $60 \%$ and $80 \%$, respectively. This image sampling redundancy is not only critical for providing a basis for checking scene illumination uniformity, as will be demonstrated in this study, but also for generating 3D point clouds, digital surface models (DSMs), and orthomosaics using UAV Structure from Motion (SfM). In particular, SfM is a photogrammetric 3D reconstruction technique that uses overlapping 2D images to create 3D point clouds, DSMs, and orthomosaics. SfM involves three stages, namely (1) feature detection, (2) image matching, and (3) bundle block adjustment (Wang et al. 2014). Furthermore, the proprietary Agisoft Photoscan (Agisoft LLC 2016) software was used for image mosaicking using UAV-SfM. The geotagged raw images were administered into the Agisoft Photoscan photogrammetry package together with $10 \mathrm{GCP}$ points to produce the georectified RGB orthomosaic as done by Coveney and Roberts (2017). SfM does not require camera exterior orientations or interior orientations but the Agisoft lens software was used for lens distortion geometric calibration (Fraser et al. 2016). The 10 GCPs were used to increase the mosaicking speed and image matching accuracy.

\subsection{Radiometric calibration results}

3.4.1. Scene illumination uniformity check experiment using the Student's $t$-test Table 2 shows the descriptive statistics used in the two sample Student's $t$-test performed between the mean DN of the reference illumination uniformity check marker

Table 2. Descriptive statistics for reference uniformity check L-shaped markers.

$\begin{array}{lcc}\text { Statistic } & \text { B01 marker } & \text { B02-B10 markers } \\ \text { Mean DN } & 223.98 & 222.62 \\ \text { Variance of DNs } & 34.63 & 28.63 \\ \text { STD deviation of DNs } & 5.88 & 5.35\end{array}$


(B01) and the other L-shaped markers (B2-B10). In particular, the reference L-shaped marker (B01) had higher variance, standard deviation, and mean pixel DNs than the overall variance, standard deviation, and mean pixel DNs of the other scene illumination uniformity check L-shaped markers. However, the difference of 1.36 between the mean DNs in Table 2 was not found to be statistically significant at the $95 \%$ confidence interval $(p<0.05)$. This information can be considered to infer that it is justifiable to calibrate the entire orthomosaic using ELM prediction equations derived from mean reflectance values measured in only one spot within the 97 ha RGB orthomosaic. These results show that the entire scene being mapped is experiencing similar atmospheric and illumination conditions.

\subsubsection{Regression of calibration target image mean DNs and mean in situ spectral reflectance}

A summary of the regression relationships between image DNs and in situ reflectance measurements in the red, green, and blue wavebands is presented in Table 3. The regression equations results show that the relationship between image DNs and in situ reflectance measurements in all the camera wavebands is exponential (Equations (2)-(4)). An exemplary visual depiction of the exponential relationship for the red waveband is shown in Figure 7. Furthermore, Figure 8 shows that there exists a linear relationship between the natural log transformed reflectance values and image DNs. A similar observation was also found in Wang and Myint (2015). As a result, the $y$-intercept 3.423

(Figure 8 and Equation (8) in Table 3) can be converted to reflectance (0.033), which represents the minimum at-surface reflectance that the Sony Nex-7 CMOS sensor used in this study can detect in the red waveband colour channel. In addition, the utilized CMOS sensor can detect minimum at-surface reflectance values of 0.032 and 0.029 for the green and blue wavebands, respectively. The minimum at-surface reflectance values for the green and blue wavebands were derived using the constants in Equations (9) and (10).

\subsubsection{Empirical line calibration equations}

They-intercept value for each linear equation represents the constant calibration parameter in the ELM calibration prediction equation of type:

$$
-\ln (y)=m \times(\mathrm{DN})+C
$$

where $y$ represents reflectance, mbeing the gradient of each image waveband in the derived ELM calibration equation, and $C$ representing the constant calibration parameter (Table 4, Equations (12)-(14)). The derived linear Equations (12)-(14) in Table 4 were used to calibrate the 97 ha RGB orthomosaic image wavebands. Subsequent layer stacking, quantitative analysis, and image classification can thus be performed on the calibrated orthomosaic following the procedures outlined by Mafanya et al. 2017 for mapping $H$. pomanensis from UAV derived ultra-high spatial resolution RGB imagery.

\subsubsection{Error analysis between estimated and in situ measured reflectance values}

To validate the proposed vicarious radiometric calibration methodological framework, reflectance values derived from the calibrated image wavebands were regressed against in situ measured reflectance values for the check targets. Results shown in Table 5 present the comparison between mean reflectance values derived from the calibrated 
Table 3. Summary of regression relationships for each waveband.

\begin{tabular}{ll} 
Regression equation & \multicolumn{1}{c}{ Red band } \\
Coefficient of determination & $y=0.032 e^{(0.014 \mathrm{DN})}$ \\
Linearized equation & $R^{2}=0.99(p<0.01)(5)$ \\
& $y=-0.014 x+3.423(8)$
\end{tabular}

$$
\begin{gathered}
\text { Green band } \\
y=0.032 e^{(0.013 \mathrm{DN})}(3) \\
R^{2}=0.99(p<0.01)(6) \\
y=-0.013 x+3.434(9)
\end{gathered}
$$

$$
\begin{gathered}
\text { Blue band } \\
y=0.029 e^{(0.013 \mathrm{DN})}(4) \\
R^{2}=0.98(p<0.01)(7) \\
y=-0.013 x+3.518(10)
\end{gathered}
$$




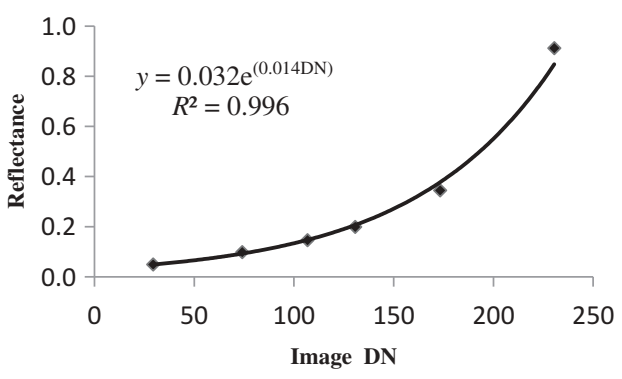

Figure 7. Example of the relationship between image digital numbers and in situ at-surface reflectance values for the red waveband.

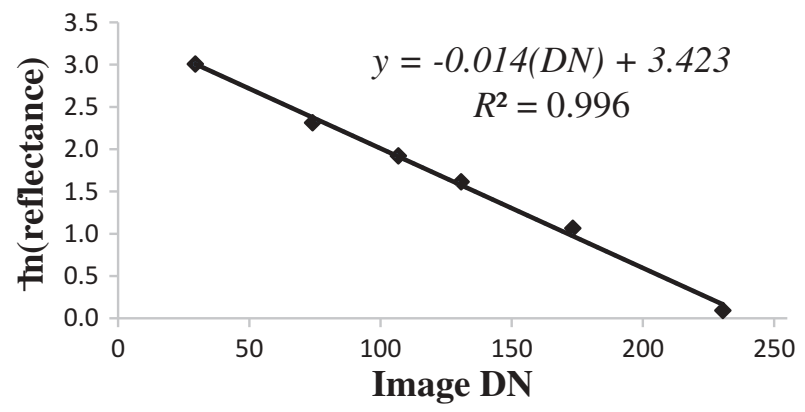

Figure 8. Example of the relationship between natural log-transformed reflectance and in situ atsurface reflectance values for the red waveband.

Table 4. Linear calibration equations for the red, green, and blue wavebands.

$\begin{array}{ll}\text { Image waveband } & \text { Radiometric calibration equation } \\ \text { Red } & -\ln (y)=-0.01413 \times(\mathrm{DN})+3.423(12) \\ \text { Green } & -\ln (y)=-0.01385 \times(\mathrm{DN})+3.434(13) \\ \text { Blue } & -\ln (y)=-0.01338 \times(\mathrm{DN})+3.518(14)\end{array}$

image wavebands (estimated) and their respective in situ at-surface reflectance values (measured) for the black, blue, green, red, and white check targets. There are three data points for each check target representing each waveband. The regression between the reflectance values derived from the calibrated image wavebands and the measured atsurface reflectance values resulted in a correlation coefficient $r$ value of $0.977(p<0.01)$ as shown in Figure 9. Furthermore, the RMSE between estimated and measured reflectance values in Table 5, i.e. for all the three wavebands and five check targets, was found to be 0.063 , whereas the waveband specific RMSE values were $0.040,0.048$, and 0.089 for the red, green, and blue image wavebands, respectively. The aforementioned strong correlation, coupled with the low overall RMSE, suggests low discrepancies between the in situ measured mean reflectance values and the reflectance values derived from the radiometric calibrated image wavebands. In particular, the highest RMSE value of 0.089 reported for the blue waveband could be attributed to the fact that scattering of 
Table 5. Comparison between check targets (black, blue, green, red, and white) mean reflectance values and their respective in situ at-surface reflectance values.

Estimated
reflectance

\section{Black square}

Red

Green

Blue

Blue circle

Red

Green

Blue

Green circle

Red

Green

Blue

Red circle

Red

Green

Blue

White square

Red

Green

Blue
0.046607

0.044089

0.041845

0.046601

0.062312

0.361492

0.088030

0.447994

0.132574

0.557448

0.070380

0.074535

0.709671

0.717760

0.658850
Measured

reflectance

0.054228

0.049955

0.047642

0.026515

0.066245

0.241355

0.150555

0.362182

0.110585

0.521022

0.070882

0.053538

0.759841

0.782323

0.815857

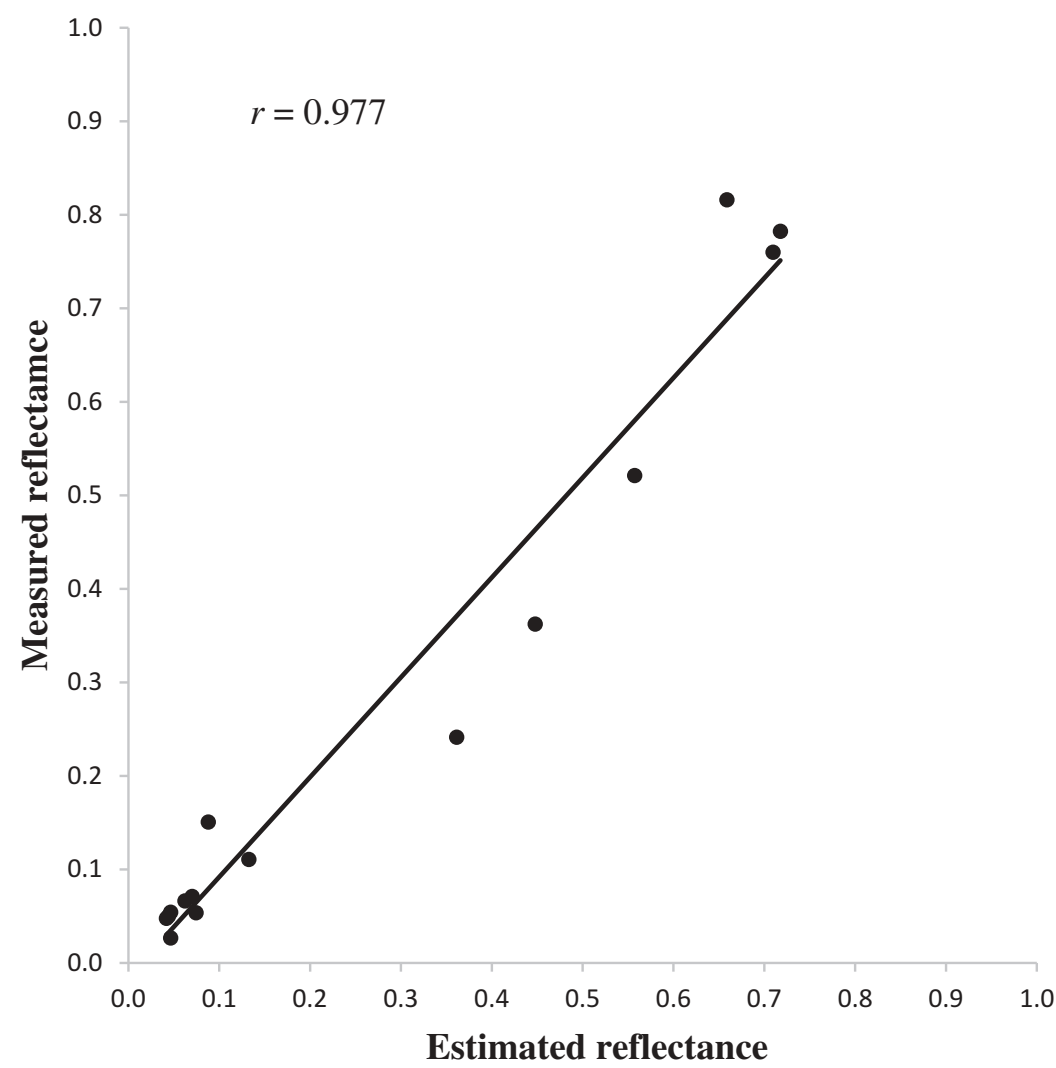

Figure 9. Regression between in situ measured reflectance and the reflectance values derived from the calibrated image wavebands $(r=0.977)$. 
radiation by the atmosphere is greater at shorter wavelengths (Smith and Milton 1999). Hence, the in situ proximally sensed white target mean reflectance of 0.816 is much higher than the remotely sensed mean reflectance of 0.659 in the blue waveband colour channel of the Sony Nex-7 camera. This is because proximal sensing was done at $1 \mathrm{~m}$ above the check targets while remotely sensed images were taken at $160 \mathrm{~m} \mathrm{AGL}$, meaning radiation interacted with more atmospheric particles in the latter case. In overall, these error analysis and the strong correlation results validate the proposed radiometric calibration framework for applications in flat terrain semi-arid woodlands.

\section{Discussion and conclusion}

Radiometric calibration methods are usually developed for single-image samples as done in Kelcey and Lucieer (2012), Del Pozo et al. (2014), and Taylor (2015). However, the tendency of IAPs to spread over large areas necessitates the development of vicarious radiometric calibration methods that can be applied on image data spanning large spatial extents and this requires large enough calibration targets. This study demonstrated a radiometric calibration framework for UAV-derived orthomosaics for application in flat semi-arid woodlands. First, it was demonstrated that the relatively affordable Masonite hardboards painted with varying grey values of black and white water paint are highly Lambertian and appropriate for use in calibration target design. This property was observed both in a controlled room with an artificial light source and on the field in the semi-arid woodland study area. The Masonite hardboards of $244 \mathrm{~cm} \times$ $122 \mathrm{~cm}$ were found to be large enough to allow painting of two grey levels in tandem configuration when the UAV is flown at an altitude of less than $160 \mathrm{~m} \mathrm{AGL}$. Some studies make use of vinyl sheet canvas to design the greyscale calibration targets as done in Del Pozo et al. 2014), and thus, the target material of choice can be influenced not only by Lambertian properties but also availability, transportability, and ease of use in different environmental settings.

Second, a scene illumination uniformity check method was developed in this study to assess whether the incoming radiant flux is uniform across the orthomosaic. The solar irradiance within the 97 ha study area was found to be uniform, thus indicating that all the 611 images taken by the Sony NEX-7 were acquired under the same environmental conditions. The hypothesis was that under the same environmental conditions the DNs of the same target material should be similar and this was tested at a $95 \%$ confidence interval. The difference of 1.36 between the mean DNs of the reference L-target and the other L-targets in Table 2 was found not to be statistically significant at the $95 \%$ confidence interval, and from this, it was inferred that it is justifiable to calibrate the entire scene using prediction equations derived using mean reflectance values measured from only a single spot within the mapped area.

Third, this study demonstrated that there exists an exponential relationship between target DNs and their corresponding at-surface mean reflectance values (Equations (2)(4)). However, in most studies, e.g. in Dean, Warner, and Mcgraw (2000), Karpouzli and Malthus (2003), Levin, Ben-Dor, and Singer (2009), and Berni et al. (2009), the empirical linear calibration equation were derived by assuming a linear relationship between image DNs and calibration targets in situ reflectance values. This assumption is usually made when there is only two calibration targets, e.g. a white and a black target (Smith 
and Milton 1999). In this study, this relationship was found to be exponential with coefficient of determination $R^{2}$ values greater than 0.98 (Equations (5)-(7)) as found in Wang and Myint (2015). Figure 7 is an example of the observed exponential relationship between image DNs and the mean in situ measured reflectance values of the calibration targets. Upon transformation of the exponential equations by taking the natural log, the constant calibration parameters of the empirical linear equations were derived. The three ELM calibration prediction equations were then derived by first computing the gradient of the prediction equation using the calibration target average DN and its average in situ reflectance as well as the constant calibration parameter or $y$-intercepts of the linear equations (Table 4).

Finally, the accuracy of at-surface reflectance values in the calibrated image wavebands was assessed using in situ spectral measurements for white, red, green, blue, and black reinforced PVC targets, resulting in a correlation coefficient $r 0.96$ $(p<0.01)$ and overall RMSE of 0.063 . These findings suggest that given the entire scene being mapped is experiencing similar irradiance flux under similar environmental conditions according to the scene illumination uniformity assessment, then radiometric calibration using mean reflectance values measured from only one area of the orthomosaic can be used to calibrate the entire orthomosaic. The proposed framework is not only important for mapping IAPs but also for use in large-scale crop mapping applications in precision agriculture, land-use/land-cover monitoring, as well as vegetation mapping and classification, particularly for mapping widespread IAPs such as $H$. pomanensis. The limitation of this research is that it has no way of showing the largest spatial coverage for which the demonstrated vicarious radiometric calibration framework could be applied. This, however, could also depend on the nature of the environment that is being mapped as well as prevailing weather conditions. Future research objectives could be to apply this framework for areas larger than 200 ha as the developments in UAV and battery technology are increasingly enabling these systems to map larger areas. Most studies that applied UAV-RS for mapping IAPs made use of thematic image classification without conducting either sensor geometric or image radiometric calibration, for instance, Dvořák et al. (2015), Müllerová et al. (2016, 2017), Hill et al. (2017), and Mafanya et al. (2017). Therefore, another future research objective would be to assess whether radiometric calibration improves the image classification accuracy for mapping and detecting various IAPs in different environmental settings. Another important research avenue is quantitative image analysis using radiometric calibrated UAV-RS imagery for improving the estimation of biophysical and biochemical constituents in plants from UAV imagery.

\section{Acknowledgments}

The authors thank Dave Cochran, Barend Swart, Phomolo Seriba, Owen Vyk, Monty Tshwale, Malherbe Rossouw, and Kutlwano Maleka for assisting with the recording of spectral data, image data acquisition process, and provision of data processing equipment. Furthermore, the authors would like to thank Eric Economon and Sedgely Pinneken for helping with the planning and implementation of spectral data acquisition. The authors would also like to thank Faizel Khan for helping with the radiometric calibration accuracy assessment. In addition, 
the authors acknowledge insights from Kuhle Siseko Ndyamboti and Mnqweno Mnyengeza for helping with the results analysis. Moreover, the authors acknowledge the three anonymous reviewers whose constructive and critical comments improved the quality of this work. Lastly, the authors acknowledge Dr Helmuth Zimmermann's guidance in identifying the woodland tree species.

\section{Author contributions}

M.M., P.T., and P.M. conceived the research idea. M.M. and P.T. conducted the data analysis, literature review, tables, figures, and preparation of manuscript. J.B.G.C and T.M. managed the preparation of the manuscript and performed editing.

\section{Disclosure statement}

No potential conflict of interest was reported by the authors.

\section{Funding}

This research work was supported by the South African National Department of Environmental Affairs through its funding of the South African National Biodiversity Institute Directorate: Biological Invasions, project number [P038].

\section{References}

Agisoft LLC. "Agisoft PhotoScan." Accessed 15 November 2016. http://www.agisoft.com/down loads/installer/

Akar, Ö. 2017. "Mapping Land Use with Using Rotation Forest Algorithm from UAV Images." International Journal of Remote Sensing 50 (1): 269-279.

Babapour, H., M. Mokhtarzade, and M. J. Valadan Zoej. 2017. "A Novel Post-Calibration Method for Digital Cameras Using Image Linear Features." International Journal of Remote Sensing 38 (8-10): 2698-2716. doi:10.1080/01431161.2016.1232875.

Bagheri, N. 2017. "Development of a High-Resolution Aerial Remote Sensing System for Precision Agriculture." International Journal of Remote Sensing 38 (8-10): 2053-2065. doi:10.1080/ 01431161.2016 .1225182$.

Berni, J. A. J., S. Member, P. J. Zarco-Tejada, L. Suárez, and E. Fereres. 2009. "Thermal and Narrowband Multispectral Remote Sensing for Vegetation Monitoring from an Unmanned AerialVehicle." IEEE Transactions on Geoscience and Remote Sensing 47 (3): 722-738.

Berra, E., S. Gibson-Poole, A. MacArthur, R. Gaulton, and A. Hamilton. 2015. "Estimation of the Spectral Sensitivity Functions of Un-Modified and Modified Commercial Off-The-Shelf Digital Cameras to Enable Their Use as a Multispectral Imaging System for UAVs." The International Archives of the Photogrammetry, Remote Sensing and Spatial Information Sciences, no. XL-1/W4: 207-214. doi:10.5194/isprsarchives-XL-1-W4-207-2015.

Bonnet, S., J. Lisein, and P. Lejeune. 2017. "Comparison of UAS Photogrammetric Products for Tree Detection and Characterization of Coniferous Stands." International Journal of Remote Sensing 38 (19): 5310-5337.

Candiago, S., F. Remondino, M. De Giglio, M. Dubbini, and M. Gattelli. 2015. "Evaluating Multispectral Images and Vegetation Indices for Precision Farming Applications from UAV Images." Remote Sens 7 (4): 4026-4047. doi:10.3390/rs70404026.

Clemens, S. R. 2012. "Procedures for Correcting Digital Camera Imagery Acquired by the AggieAir Remote Sensing Platform." All Graduate Plan B and other Reports. Paper 186.1-59. 
Coveney, S., and K. Roberts. 2017. "Lightweight UAV Digital Elevation Models and Orthoimagery for Environmental Applications: Data Accuracy Evaluation and Potential for River Flood Risk Modelling." International Journal of Remote Sensing 38 (8-10): 3159-3180. doi:10.1080/ 01431161.2017 .1292074$.

Cracknell, A. P. 2017. "UAVs: Regulations and Law Enforcement." International Journal of Remote Sensing 38 (8-10): 3054-3067. doi:10.1080/01431161.2017.1302115.

Crusiol, L. G. T., M. R. Nanni, G. F. C. Silva, R. H. Furlanetto, A. A. Gualberto, A. C. Gasparotto, and M. N. De Paula. 2017. "Semi Professional Digital Camera Calibration Techniques for Vis/NIR Spectral Data Acquisition from an Unmanned Aerial Vehicle." International Journal of Remote Sensing 38 (8-10): 2717-2736. doi:10.1080/01431161.2016.1264032.

Dean, C., T. A. Warner, and J. B. Mcgraw. 2000. "Suitability of the DCS460c Colour Digital Camera for Quantitative Remote Sensing Analysis of Vegetation." Science 55 (2): 105-118.

Del Pozo, S., P. Rodríguez-Gonzálvez, D. Hernández-López, and B. Felipe-García. 2014. "Vicarious Radiometric Calibration of a Multispectral Camera on Board an Unmanned Aerial System." Remote Sens 6: 1918-1937.

Dvořák, P., J. Müllerová, T. Bartaloš, and J. Brůna. 2015. “Unmanned Aerial Vehicles for Alien Plant Species Detection and Monitoring." The International Archives of the Photogrammetry, Remote Sensing and Spatial Information Sciences XL-1/W4: 83-90. doi:10.5194/isprsarchives-XL-1-W4-83-2015.

Femondimo, F., L. Barazzetti, F. Nex, M. Scaioni, and D. Sarazzi. 2011. "UAV Photogrammetry for Mapping and 3D Modelling- Current Status and Future Perspectives." The International Archives of the Photogrammetry, Remote Sensing and Spatial Information Sciences XXXVIII-1/ C22: 25-30.

Fernández-Hernandez González-Aguiler, D., P. Rodriguez-Gonzalvez, and J. Mancera-Taboada. 2015. "Image-Based Modelling from Unmanned Aerial Vehicle (UAV) Photogrammetry: An Effective Low-Cost Tool for Archeological Applications." Archeometry 57 (1): 128-145. doi:10.1111/arcm.12078.

Fraser, R. H., I. Olthof, T. C. Lantz, and C. Schmitt. 2016. "UAV Photogrammetry for Mapping Vegetation in Low-Artic." Arctic Science 2: 79-102. doi:10.1139/as-2016-0008.

Gómez-Candón, D., A. I. De Castro, and F. Lopez-Granados. 2014. "Assessing the Accuracy of Mosaics from Unmanned Aerial Vehicle (UAV) Imagery for Precision Agriculture Purposes in Wheat." Precision Agricultural 15: 44-56. doi:10.1007/s11119-013-9335-4.

Grenzdörffer, G. J., A. Engel, and B. Teichert. 2008. "The Photogrammetric Potential of Low-Cost UAVs in Forestry and Agriculture." The International Archives of the Photogrammetry, Remote Sensing and Spatial Information Sciences XXXVII (B1): 1207-1214.

Haghighattalab, A., L. González Pérez, S. Mondal, D. Singh, D. Schinstock, J. Rutkoski, I. OrtizMonasterio, P. Singh, D. Goodin, and J. Poland. 2016. "Application of Unmanned Aerial Systemsfor High Throughput Phenotyping of Large Wheat Breeding Nurseries." Plant Methods 12: 35. doi:10.1186/s13007-016-0115-9.

Hakala, T., J. Suomalainen, and J. I. Peltoniemi. 2010. "Acquisition of Bidirectional Relectance Factor Dataset Using a Micro Unmanned Aerial Vehicle and a Consumer Camera." Remote Sens 2 (3): 819-832. doi:10.3390/rs2030819.

Hill, D. J., C. Tarasoff, G. E. Whitworth, J. Baron, J. L. Bradshaw, and J. S. Church. 2017. "Utility of Unmanned Aerial Vehicles for Mapping Invasive Plant Species: A Case Study on Yellow Flag Iris (Iris pseudacorus L.)." International Journal of Remote Sensing 38 (8-12): 2083-2105. doi:10.1080/ 01431161.2016.1264030.

Honkavaara, E., H. Saari, J. Kaivosoja, I. Pölönen, T. Hakala, P. Litkey, J. Mäkynen, and L. Pesonen. 2013. "Processing and Assessment of Spectrometric, Stereoscopic Imagery Collected Using a Lightweight UAV Spectral Camera for Precision Agriculture." Remote Sens 5: 5006-5039. doi:10.3390/rs5105006.

Honkavaara, E., R. Arbiol, L. Markelin, L. Martinez, M. Cramer, S. Bovet, L. Chandelier, et al. 2017. "Digital Airborne Photogrammetry-A New Tool for Quantitative Remote Sensing?-A State-Ofthe-Art Review on Radiometric Aspects of Digital Photogrammetric Images." Remote Sensing 1: 577-605. doi:10.3390/rs1030577. 
Hugemann, W. 2010. Correcting Lens Distortions in Digital Photographs, 1-12. Leverkusen, Germany: Ingenieurbüro Morawski + Hugemann.

Karpouzli, E., and T. Malthus. 2003. "The Empirical Line Method for the Atmospheric Correction of IKONOS Imagery." International Journal Remote Sensing 24 (5): 1143-1150. doi:10.1080/ 0143116021000026779.

Kelcey, J., and A. Lucieer. 2012. "Sensor Correction and Radiometric Calibration of a 6-Band Multispectral Imaging Sensor for UAV Remote Sensing." ISPRS - Archives XXXIX-B1: 393-398.

Laliberte, A. S., M. Goforth, C. M. Steele, and A. Rango. 2011. "Multispectral Remote Sensing from Unmanned Aircraft: Image Processing Workflows and Applications for Rangeland Environments." Remote Sensing 3 (11): 2529-2551. doi:10.3390/rs3112529.

Levin, E., E. Ben-Dor, and A. Singer. 2009. "A Digital Camera as Tool to Measure Colour Indices and Related Properties of Sandy Soils in Semi-Arid Environments." International Journal of Remote Sensing 26 (24): 5475-5492. doi:10.1080/01431160500099444.

Li, W., Z. Niu, H. Chen, and D. Li. 2017. "Characterizing Canopy Structural Complexity for the Estimation of Maize LAI Based on ALS Data and UAV Stereo Images." International Journal of Remote Sensing 38 (8-10): 2106-2116. doi:10.1080/01431161.2016.1235300.

Mafanya, M., P. Tsele, J. Botai, P. Manyama, B. Swart, and T. Monate. 2017. "Evaluating Pixel and Object Based Image Classification Techniques for Mapping Plant Invasions from UAV Derived Aerial Imagery: Harrisia pomanensis as a Case Study." ISPRS Journal of Photogrammetry and Remote Sensing 129: 1-11. doi:10.1016/j.isprsjprs.2017.04.009.

Müllerová, J., J. Brůna, P. Dvořák, T. Bartaloš, and M. Vítková. 2016. "Does the Data Resolution/ Origin Matter? Satellite, Airborne and UAV Imagery to Tacle Plant Invasions." The International Archives of the Photogrammetry, Remote Sensing and Spatial Information Sciences XLI-B7: 903908. doi:10.5194/isprsarchives-XLI-B7-903-2016.

Müllerová, J., T. Bartaloš, J. Brůna, P. Dvořák, and M. Vítková. 2017. “Unmanned Aircraft in Nature Conservation: An Example from Plant Invasions." International Journal of Remote Sensing 38 (810): 2177-2198. doi:10.1080/01431161.2016.1275059.

Mzezwa, J., T. Mlisi, and L. Van Rensburg. 2010. “Characterisation of Rainfall at a Semi-Arid Ecotope in the Limpopo Province (South Africa) and Its Implications for Sustainable Crop Production." Water SA 36 (1): 19-26.

Nguyen, H. C., J. Jung, J. Lee, S. U. Choi, S. Y. Hong, and J. Heo. 2015. “Optimal Atmospheric Correction for Above-Ground Forest Biomass Estimation with the ETM+ Remote Sensor." Sensors 15: 18865-18886. doi:10.3390/s150818865.

Osborne, M. "Ardupilot Mission Planner." Accessed 10 August 2016. http://planner.ardupilot.com/

Pádua, L., J. Vanko, J. Hruška, T. Adão, J. J. Sousa, E. Peres, and R. Morais. 2017. "UAS, Sensors, and Data Processing in Agroforestry: A Review Towards Practical Applications." International Journal of Remote Sensing 38 (8-10): 2349-2391. doi:10.1080/01431161.2017.1297548.

Primicerio, J., S. F. Di Gennaro, E. Fiorillo, L. Genesio, E. Lugato, A. Matese, and F. P. Vaccari. 2012. “A Flexible Unmanned Aerial Vehicle for Precision Agriculture." Precision Agricultural 13 (4): $517-$ 523. doi:10.1007/s11119-012-9257-6.

Quantum GIS development team. 2017. Quantum GIS Geographic Information System. Open Source Geospatial Foundation Project. http://qgis.osgeo.org

Rinaudo, F., F. Chiabrando, A. Lingua, and A. Spano. 2012. "Archeological Site Monitoring: UAV Photogrammetry Can Be an Answer. The International Archives of the Photogrammetry." Remote Sensing and Spatial Information Sciences XXXXIX-B5: 583-588.

Rokhmana, C. K. 2015. "The Potential of UAV-based Remote Sensing for Supporting Precision Agriculture in Indonesia." Procedia Environmental Sciences 24: 245-253. doi:10.1016/j. proenv.2015.03.032.

Smith, G. M., and E. J. Milton. 1999. "The Use of the Empirical Line Method to Calibrate Remotely Sensed Data to Reflectance." International Journal of Remote Sensing 20 (13): 2653-2662. doi:10.1080/014311699211994.

Sonaa, G., D. Passonia, L. Pintoa, D. Pagliaria, D. Masseroni, B. Ortuanib, and A. Facchib. 2016. "UAV Multispectral Survey to Map Soil and Crop for Precision Farming Applications." The International 
Archives of the Photogrammetry, Remote Sensing and Spatial Information Sciences XLI-B1: 1023-1029. doi:10.5194/isprsarchives-XLI-B1-1023-2016.

Staben, G. W., K. Pfitzner, R., . Bartolo, and A. Lucieer. 2012. "Empirical Line Calibration of WorldView-2 Satellite Imagery to Reflectance Data: Using Quadratic Prediction Equations." Remote Sensing Letters 3 (6): 521-530. doi:10.1080/01431161.2011.609187.

Taylor, C. 2015. Radiometric Calibration of a Modified DSLR for NDVI, 1-7. Rochester Institute of Technology. https://www.cis.rit.edu/ cnspci/references/theses/senior/taylor2015.pdf

Tesfamichael, S. G., S. W. Newete, E. Adamd, and B. Dubula. 2017. "Field Spectroradiometer and Simulated Multispectral Bands for Discriminating Invasive Species from Morphologically Similar Cohabitant Plants." GIScience \& Remote Sensing 55 (3): 1548-1603. doi:10.1080/ 15481603.2017.1396658.

Thiel, C., and C. Schmullius. 2017. "Comparison of UAV Photograph-Based and Airborne LidarBased Point Clouds over Forest from a Forestry Application Perspective." International Journal of Remote Sensing 38 (8-10): 2411-2426. doi:10.1080/01431161.2016.1225181.

Torrescan, C., A. Berton, F. Carotenuto, S. F. Di Gennaro, B. Gioli, A. Matese, F. Miglietta, C. Vagnoli, A. Zaldei, and L. Wallace. 2017. "Forestry Applications of UAVs in Europe: A Review." International Journal of Remote Sensing 38 (8-10): 2427-2447. doi:10.1080/01431161.2016.1252477.

Wang, C., and S. W. Myint. 2015. "A Simplified Empirical Line Method of Radiometric Calibration for Small Unmanned Aircraft Systems-Based Remote Sensing." IEEE J-STARS 8: 1-10.

Wang, J., F. Shi, J. Zhang, and Y. Liu 2006. "A New Calibration Model and Method of Camera Lens Distortion." Proceedings of the 2006 IEEE/RSJ International Conference on Intelligent Robots and Systems. Beijing, China, October 9-15.

Wang, Q., L. Wu, S. Chen, D. Shu, Z. Xu, F. Li, and R. Wang. 2014. "Accuracy Evaluation of 3D Geometry from Low-Attitude UAV Images: A Case Study at Zijin Mine." ISPRS - Archives XL-4: 297-300.

Wilson, J. R., F. D. Panetta, and C. Lindgren. 2016. Detecting and Responding to Alien Plant Incursions. Cambridge, UK: Cambridge University Press.

Wilson, J. R., P. Ivey, and Nanni I. 2013 "A New National Unit for Invasive Species Detection, Assessment and Eradication Planning". South African Journal of Science 109 (5): 61-13.

Yang, G., J. Liu, C. Zhao, Z. Li, Y. Haung, H. Yu, B. Xu, et al. 2017. “Unmanned Aerial Vehicle Remote Sensing for Field-Based Crop Phenotyping: Current Status and Perspectives." Frontiers Plant Sciences 8: 1111. doi:10.3389/fpls.2017.01111.

Zhang, Z. X., "Native Vegetation Mapping Classification Using Remote Sensing Techniques: A Case Study of Dairy Flat Regrowth Bush by Using the AUT Unmanned Aerial Vehicle." Auckland University of Technology thesis, 2014. doi:10.1128/AEM.03322-14 